\title{
UTILIZAÇÃO DA BIOMASSA DE Artemia franciscana COMO ADITIVO ALIMENTAR NO CULTIVO LABORATORIAL DO CAMARÃO MARINHO Litopenaeus schmitti
}

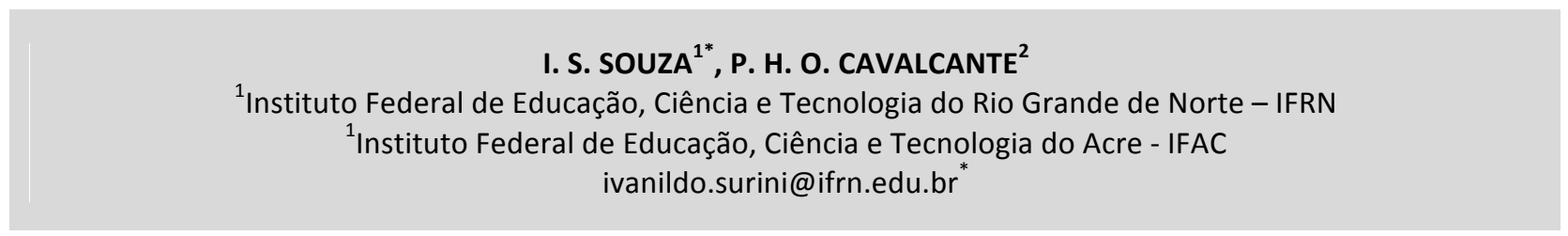

Submetido 14/04/2016 - Aceito 20/04/2018

DOI: $10.15628 /$ holos.2018.4427

\section{RESUMO}

O presente trabalho tem por objetivo avaliar a utilização da biomassa de Artemia franciscana como aditivo alimentar no cultivo laboratorial do camarão marinho Litopenaeus schmitti. Durante 30 dias, os camarões $(n=20)$ foram alimentados com ração comercial revestida com 3 diferentes níveis de biomassa de $A$. franciscana, correspondentes a 1, 3 e $5 \%$ da quantidade de ração ofertada. As variáveis temperatura, $\mathrm{pH}$, salinidade, luminosidade, $\mathrm{NH}_{3}$ e $\mathrm{NO}_{2}^{-}$da água foram monitoradas. Como critérios de avaliação foram determinados o ganho de peso absoluto (GPA), o ganho de peso relativo (GPR), a taxa de crescimento relativo (TCR) e o consumo alimentar aparente (CAA) dos camarões. Adicionalmente, a estabilidade da ração física da ração e o teor de umidade da biomassa de $A$. franciscana foram aferidos. Os resultados relativos à estabilidade física da ração demonstraram que passadas $2 \mathrm{~h}$ de imersão, $80,9 \pm 0,39 \%$ de seu conteúdo permaneceu íntegro. Por sua vez, o teste de umidade da biomassa indicou que $7,49 \pm 0,19 \%$ de peso de $A$. franciscana representa a massa corpórea que revestiu as partículas de ração. Ao fim do experimento, o desempenho dos animais quanto ao GPA, GPR e TCR foi melhor nos tratamentos aditivados com maior percentual de biomassa. Já o CAA não apresentou diferenças estatisticamente significativas $(P>0,05)$ nos diferentes tratamentos. Os resultados desse estudo indicam que a adição de biomassa congelada de $A$. franciscana adulta à rações comerciais, em níveis correspondentes a 1, 3 e 5\% da oferta diária de ração, aumenta o ganho de peso de juvenis de L. schmitti.

PALAVRAS-CHAVE: Aditivo alimentar; Artemia; camarão marinho.

\section{USE OF BIOMASS Artemia franciscana AS A FOOD ADDITIVE IN THE LABORATORY CULTIVATION OF MARINE SHRIMP Litopenaeus schmitti}

\begin{abstract}
The objective of the current study is to evaluate the use of adult Artemia franciscana biomass as a feeding additive in the laboratorial culture of the autochthonous species of marine shrimp Litopenaeus schmitti. For 30 days, shrimp $(n=20)$ were fed a commercial ration coated with three different levels of $A$. franciscana biomass corresponding to 1,3 , and $5 \%$ of the daily feed offer. In water, the variables temperature, $\mathrm{pH}$, salinity, luminosity $\mathrm{NH}_{3}, \mathrm{NO}_{2}^{-}$were measured. The absolute weight gain (GPA), the relative weight gain (GPR), the relative growth rate (TCR), and the apparent feed consumption (CAA) of shrimp were used as evaluation criteria. In addition, the physical stability of ration in water after 2 hours and the water content of $A$. franciscana biomass were measured. The stability of the
\end{abstract}

ration in water was $80.9 \pm 0.39 \%$. On the other hand, the water content test indicated that $7.49 \pm 0.19 \%$ of the $A$. franciscana biomass represented the body mass which effectively coated the feed pellets. The results obtained for the CAA did not show significant differences ( $P$ $>0.05)$ among treatments. However, at the termination of the experiment, the growth performance (GPA, GPR, and TCR) of shrimp in the dietary treatments coated with $A$. franciscana was significantly better than animals in the control group. In conclusion, the results of the current study indicate that the coating of commercial rations with adult $A$. franciscana biomass in levels corresponding to 1,3 , and $5 \%$ of the daily feed offered, increases the weight gain of juvenile L. schmitti.

KEYWORDS: Feeding additive; Artemia; marine shrimp. 


\section{INTRODUÇÃO}

O Estado do Rio Grande do Norte é um dos maiores produtores de camarão marinho do Brasil. Com efeito, as 30.807 toneladas despescadas em 6.281 hectares de viveiros de camarão já representou $40,5 \%$ da produção e $37,8 \%$ da área de cultivo brasileiras na década passada (ABCC, 2015; RODRIGUES, 2005). Esse sucesso se deu devido principalmente ao domínio das técnicas de produção de pós-larvas em laboratório, à existência de condições ambientais favoráveis e de terras costeiras de baixo custo, à disponibilidade de mão-de-obra barata e, por último, a um alto retorno econômico (NUNES; GESTEIRA; GODDARD. 1997).

Atualmente, Litopenaeus vannamei (Boone, 1931) é a espécie predominante de camarão marinho cultivada comercialmente no Nordeste do Brasil. Essa espécie foi introduzida na década de 80 e desde então tem obtido grande sucesso e exibido elevados índices de produção. No entanto, por tratar-se de uma espécie alóctone, o cultivo de L. vannamei representa ameaças ambientais que poderão resultar na exclusão de espécies nativas através da competição ou predação, na alteração do estoque genético pelo cruzamento entre os camarões cultivados e autóctones (formação de híbridos) e na introdução e disseminação de agentes patogênicos que afetem os estoques selvagens (WESTON 1991; PRIMAVERA 1993; MISAMORE; BROWDY 1997; NAYLOR et al. 2000; NAYLOR; WILLIANS, STRONG, 2001). Diante dessa problemática, urge a realização de pesquisas que visem à substituição de $L$. vannamei por espécies autóctones de camarão. Nesse sentido, Litopenaeus schmitti (Burkenroad, 1936) enquadra-se como um potencial candidato, uma vez que está amplamente distribuído no extenso litoral brasileiro, além de possuir um positivo histórico produtivo nos primórdios da carcinocultura no país (BUENO, 1990).

Para alcançar o atual sucesso produtivo, os camarões passaram a ser cultivados em elevadas densidade de estocagem, existindo dessa forma a necessidade de introduzir alimentos artificiais (ração) para incrementar a produção além dos níveis suportados pela produtividade natural das unidades de cultivo. Isso gera uma outra grande preocupação, já que as rações, apesar de nutricionalmente balanceadas, muitas vezes são ineficazes e proporcionam um baixo consumo por parte dos camarões, por apresentarem reduzida atratibilidade, palatibilidade e/ou digestibilidade. Com isso, resíduos provenientes da ração não consumida rapidamente serão liberados e acumular-se-ão ao longo do tempo nas unidades de cultivo e no seu entorno, as quais poderão ter a qualidade da água e do substrato deteriorada (SMITH et al. 2002). Somado a isso, doenças, estresse e o baixo crescimento dos camarões também podem ser citados como resultado da reduzida ingestão do alimento (MARTINEZ-CORDOVA et al. 2002).

Assim, a necessidade de reduzir os desperdícios de ração e de otimizar as taxas de conversão alimentar tem sido apontada como um dos pontos mais importantes para diminuir os custos de produção de empresas que cultivam camarões, uma vez que os gastos com alimentação representam 40 a 60\% dos seus custos operacionais (MONTEMAYOR et al. 2005).

O termo aditivo alimentar compreende todas as substâncias atrativas e/ou estimulantes capazes de provocar nos animais, respectivamente, uma orientação e deslocamento em direção à fonte de alimento, e a continuidade da alimentação após tê-los iniciada (LEE; MEYERS, 1996). Portanto, presume-se que os aditivos atuem à distância (relacionado à olfação do animal e a atratibilidade da ração) ou quando os camarões estejam em contato direto com o alimento 
(relacionado à gustação do animal e a palatibilidade da ração). Nesse sentido, o uso de atrativos e estimulantes alimentares adicionados às dietas assume relevância, já que estes podem provocar, respectivamente, um rápido deslocamento dos camarões até a ração (fonte atrativa) e sua rápida ingestão. Isso implica em melhor eficiência alimentar dos animais e, consequentemente, reduz o custo global por unidade de produção. Ainda como resultado dessa rápida ingestão, o potencial para a liberação e lixiviação de nutrientes no meio será amortizado (SANCHEZ et al. 2005). Finalmente, os aditivos (atrativos e estimulantes) alimentares poderão ser usados para aumentar as taxas de crescimento, as quais também se manifestam como um dos fatores críticos para o progresso das fazendas industriais de camarão (NEW 1976; HEINEN, 1980; LEE; MEYERS, 1996; HARPAZ, 1997; FELIX; SUDHARSAN, 2004).

Nessas circunstâncias, suplementar alimentos artificiais com substâncias provenientes de insumos de baixo custo, encontrados em ampla disponibilidade e que tenham perfil nutricional e propriedades quimioatrativas e/ou quimioestimulantes adequados para acelerar o consumo alimentar e o crescimento de camarões, torna-se imprescindível para otimizar a sustentabilidade econômica das fazendas comerciais de camarão marinho e diminuir o custo ambiental vinculado à carcinocultura.

Estudos sobre a eficácia de aditivos alimentares adicionados à ração relatam que substâncias de baixo peso molecular, particularmente, aminoácidos (taurina, glicina, arginina, alanina), nucleotídeos e betaína, provenientes de componentes orgânicos, são identificadas como poderosos efetores químicos que promovem uma rápida ingestão do alimento, ocasionando assim um maior crescimento dos camarões (HEINEN, 1980; COSTA-PIERCE; LAWS, 1985; CARR, 1988; COSTERO; MEYERS, 1993; LEE; MEYERS, 1996; PENAFLORIDA; VIRTANEN, 1996; PITTET; ELLIS; LEE, 1996; HARPAZ, 1997). Esses componentes são muito mais efetivos em combinação do que quando usados individualmente (HEINEN, 1980). Boa parte deles é de origem marinha e encontrada, principalmente, em extratos e farinhas produzidas a partir de peixes, moluscos ou crustáceos (por exemplo, farinhas e solúveis de peixe, farinha e óleo de lula e farinha da cabeça de camarão) (COSTERO; MEYERS, 1993). No entanto, esses ingredientes são caros, escassos e variam quanto à disponibilidade ano a ano (MARTINEZ-CORDOVA et al. 2002).

O gênero Artemia (Crustacea, Anostraca) - um complexo de espécies bissexuais e partenogenéticas definidas pelo critério de isolamento reprodutivo - ocorre em ambientes hipersalinos, especialmente em lagos salgados interiores e salinas costeiras (LENZ; BROWNE, 1991). Cistos (embriões em diapausa) e biomassa (indivíduos juvenis e adultos) de Artemia são coletados nesses ecossistemas, processados e utilizados na aquicultura como alimento para uma enorme variedade de peixes e crustáceos (SORGELOOS et al., 1986; SORGELOOS et al., 1988; TACKAERT; SORGELOOS, 1991; SORGELOOS et al., 2001).

Embora os náuplios eclodidos a partir de embriões encistados continuem a representar a forma mais usual de consumo na indústria da aquicultura, a biomassa de Artemia tem assumido um papel crescentemente importante na dieta de vários organismos aquáticos (SORGELOOS et al., 2001). Exemplos clássicos da utilização de biomassa de Artemia ocorrem na China e no Brasil. Com efeito, Tackaert \& Sorgeloos (1991) registram consumo de biomassa de Artemia superior a 3.000 toneladas anuais nos cultivos de Fenneropenaeus chinensis realizados na baía de Bohay. No Brasil, mais de 240 toneladas de biomassa de Artemia franciscana - espécie dominante na América do Sul, América do Norte e Caribe (CAMARA, 2001) - são coletadas anualmente na região salineira do Rio Grande do Norte e utilizadas como complemento alimentar 
nos tanques berçários, viveiros de produção, larviculturas e laboratórios de maturação de $L$. vannamei (CAMARA, 2003; CAMARA et al., 2004).

À semelhança dos náuplios, a biomassa de Artemia apresenta perfil nutricional rico em aminoácidos essenciais, ácidos graxos altamente insaturados, hormônios, pigmentos e vitaminas, fato que justifica sua inclusão entre as principais fontes de substâncias atrativas e estimulantes alimentares para uso na aquicultura (SEIDEL; KRYZNOWEK; SIMPSON, 1980; LÉGER et al., 1986; SORGELOOS et al., 1986; KOLKOVSKI; KOVEN; TANDLER, 1997; COUTTEAU et al. 2000).

Considerando que (i) o camarão autóctone L. schmitti é uma espécie potencialmente apta à criação em cativeiro e seu cultivo minimiza vários riscos ambientais; que (ii) a adição de atrativos e estimulantes alimentares à ração melhora a eficiência alimentar dos camarões; e finalmente, que (iii) a biomassa adulta de $A$. franciscana constitui fonte de substâncias atrativo-estimulantes alimentares; o presente trabalho tem por objetivo avaliar a utilização da biomassa de $A$. franciscana como aditivo alimentar no cultivo em escala laboratorial da espécie autóctone de camarão marinho L. schmitti. Especificamente, pretende-se determinar o ganho de peso dessa espécie em cultivo que utilize ração comercial como substrato para absorção das substâncias potencialmente atrativas e estimulantes alimentares presentes na biomassa de $A$. franciscana.

\section{MATERIAIS E MÉTODOS}

\subsection{Origem dos organismos experimentais}

Juvenis de camarões marinhos da espécie autóctone $L$. schmitti foram coletados no canal de abastecimento de uma fazenda de camarão, localizada no sítio São Félix, município de Tibau do Sul - RN (6o 11' 12" S; 35 05' 31" W), às margens da Lagoa de Guaraíras. Após a coleta, os camarões foram devidamente acondicionados em reservatórios de $100 \mathrm{~L}$ contendo água do mar aerada artificialmente e transportados ao laboratório. Antes da realização do experimento, os camarões foram submetidos a um período de $96 \mathrm{~h}$ de aclimatação para ajustamento às condições laboratoriais.

\subsection{Sistema experimental}

O experimento foi conduzido em um sistema composto por 20 recipientes plásticos transparentes, de forma retangular, com volume útil de $2,5 \mathrm{~L}$ e $308 \mathrm{~cm}^{2}$ de área. Para assegurar as concentrações de oxigênio dissolvido em condições favoráveis à sobrevivência e ao crescimento dos camarões, pontos individuais de aeração foram instalados nos recipientes de cultivo. 0 fotoperíodo, $12 \mathrm{~h}$ claro/12h escuro, foi mantido através de iluminação artificial fluorescente.

\subsection{Preparação da dieta e delineamento experimental}

Os tratamentos dietéticos constituíram-se da adição de biomassa de A. franciscana adulta a uma ração comercial formulada para a engorda de juvenis do camarão marinho L. vannamei. $O$ objetivo da adição de biomassa de $A$. franciscana aos "peletes" de ração foi revesti-los com macro 
e micronutrientes, especialmente aminoácidos, dado seu papel como aditivo alimentar (HINDLEY, 1975). Com efeito, a biomassa de Artemia apresenta perfil rico em aminoácidos, inclusive aqueles utilizados usualmente como atrativos e estimulantes alimentares (LAVENS; SORGELOOS, 1996).

O desenho experimental dispunha de 4 tratamentos dietéticos e 5 réplicas. Os tratamentos consistiram no revestimento dos "peletes" de ração ofertados com três níveis de adição de biomassa congelada de $A$. franciscana adulta (correspondentes a 1, 3 e 5\% da quantidade de ração ofertada) designados, respectivamente, como tratamentos T1, T2 e T3. Adicionalmente, foi incluído um grupo controle tratado exclusivamente com ração (T4). Antes do revestimento, a biomassa de $A$. franciscana foi primeiramente descongelada e liquefeita (velocidade de 27.000 rpm) com auxílio de um homogeneizador mecânico. Em seguida, a biomassa liquefeita foi transferida para um béquer e pesada em balança digital de precisão. Posteriormente, procedeu-se com a mistura, umedecimento e revestimento homogêneo dos "peletes" de ração com a biomassa liquefeita para, somente então, dividir a dieta em porções correspondentes a cada tratamento e, subsequentemente, ofertá-las aos camarões. Este procedimento sempre ocorria antes de cada oferta alimentar.

\subsection{Manejo experimental}

Vinte camarões juvenis foram medidos (comprimento médio inicial de 101,16 $\pm 8,37 \mathrm{~mm}$ ) e pesados individualmente (peso médio inicial de $8,55 \pm 1,83 \mathrm{~g}$ ) e, em seguida, distribuídos aleatoriamente (um indivíduo por recipiente de cultivo) no sistema experimental. Verificou-se que não houve diferenças estatisticamente significativas na pesagem inicial dos indivíduos pertencentes aos diferentes tratamentos dietéticos. Tal como preconizado por D’Abramo \& Castell (1997), o cultivo individual teve o propósito de evitar possíveis influências de interações comportamentais intra-específicas que pudessem deprimir o crescimento dos organismos experimentais. Os camarões foram aclimatados para ajustamento e condicionamento (alimentação artificial) às condições laboratoriais, e substituídos, caso houvesse inanição ou morte. No decorrer do período experimental não houve pesagens para ajustes na taxa de alimentação, uma vez que isso poderia ocasionar estresse e/ou perda de apêndices estruturais que, deste modo, retardariam o crescimento dos camarões. Após 30 dias de cultivo, os animais foram novamente pesados para se obter os valores finais para o ganho de peso e taxas de crescimento.

No decorrer dos 30 dias, os camarões foram alimentados com quantidade de ração equivalente a $10 \%$ do seu peso médio inicial $(0,8 \mathrm{~g})$ em duas porções diárias, uma às $8 \mathrm{~h}(50 \%)$ e outra às $15 \mathrm{~h}$ (50\%). Imediatamente antes de cada oferta, as rações foram revestidas com biomassa de $A$. franciscana conforme os níveis de adição correspondentes a cada tratamento dietético $(0,6,1,8$ e 3,0 mg). No caso dos indivíduos pertencentes ao grupo controle, a alimentação ocorreu apenas com quantidade de ração equivalente a $10 \%$ do peso médio inicial dos camarões $(0,8 \mathrm{~g})$. Duas horas após cada arraçoamento, os restos alimentares foram removidos por sifonação para manter a qualidade da água favorável à sobrevivência e crescimento dos animais. Os restos alimentares coletados foram armazenados em frascos plásticos para posterior determinação da taxa de consumo alimentar aparente. Resíduos, fezes e, ocasionalmente, carapaças também foram diariamente removidos. 
Houve uma taxa de renovação diária de $50 \%$ do volume de água do mar filtrada $2 \mathrm{~h}$ após a última alimentação com a finalidade de manter sua qualidade em níveis satisfatórios. Toda água do mar usada para repor o volume perdido durante a renovação foi filtrada através de uma rede de zooplâncton com malha de $120 \mu \mathrm{m}$. Somado a isso, a qualidade da água dos recipientes experimentais foram monitoradas três vezes por semana quanto à temperatura, $\mathrm{pH}$, salinidade e luminosidade, e semanalmente quanto às concentrações de nitrogênio amoniacal $\left(\mathrm{NH}_{3}\right)$ e nitrito $\left(\mathrm{NO}_{2}{ }^{-}\right)$.

\subsection{Critérios de avaliação}

A sobrevivência dos camarões foi apenas monitorada durante o estudo e aferida ao fim do experimento. Para avaliar o desempenho zootécnico dos juvenis de $L$. schmitti nos diferentes tratamentos dietéticos foram usados os seguintes critérios: ganho de peso absoluto (GPA), ganho de peso relativo (GPR), taxa de crescimento relativo (TCR) e consumo alimentar aparente (CAA). Adicionalmente, a estabilidade da ração comercial após $2 \mathrm{~h}$ de imersão em água e o teor de umidade da biomassa de $A$. franciscana foram aferidos.

\subsubsection{Ganho de Peso Absoluto (GPA)}

O GPA referiu-se ao aumento da biomassa dos camarões em gramas, dado pela diferença entre o peso médio ao fim do experimento e o peso médio no início do experimento em cada tratamento.

\subsubsection{Ganho de Peso Relativo (GPR)}

O GPR foi calculado a partir da equação: $G P R=[(P f-P i / P i) \times 100]$, onde $P f$ representa a média do peso final dos camarões em cada tratamento, e $P i$ corresponde à média do peso inicial. $O$ resultado obtido foi expresso em taxa percentual (\%).

\subsubsection{Taxa de Crescimento Relativo (TCR)}

A TCR, expressa em percentual diário $\left(\% \cdot d i a^{-1}\right)$, foi calculada a partir da equação: $T C R=$ $[(L n P f-L n P i) / t] \times 100)$, onde $\operatorname{LnPf}$ indica o logaritmo natural do peso dos camarões ao fim do período experimental, LnPi corresponde o logaritmo natural do peso inicial e $t$ o tempo de duração do experimento (CAMARA, 1994).

\subsubsection{Consumo Alimentar Aparente (CAA)}

Para aferir a eficiência alimentar dos camarões em resposta às quantidades de aditivo alimentar ofertadas, utilizou-se a seguinte equação, modificada de Smith et al. (2005): CAA = [(Pf$\operatorname{Pr})] / B M \times t] \times 100$, onde $P f$ indica o peso seco da ração ofertada, $\operatorname{Pr}$ corresponde ao peso seco da ração recuperada após $2 \mathrm{~h}$ de imersão em água, $B M$ representa a biomassa média encontrada em cada recipiente de cultivo e $t$, o tempo experimental em dias. O resultado obtido foi expresso em \%BM.dia ${ }^{-1}$ (SMITH et al. 2005). 


\subsubsection{Teste de Estabilidade}

A ração comercial foi avaliada quanto à estabilidade física após 2 h de imersão em água. Para isso, três recipientes de alumínio (capacidade $100 \mathrm{~mL}$ ), após secagem em estufa $(55$ 으) por 60 min e posterior aferição de seu peso seco, receberam aproximadamente $1 \mathrm{~g}$ de ração. Em seguida, $40 \mathrm{~mL}$ de água destilada foram colocadas nos recipientes. Após as $2 \mathrm{~h}$ de imersão, o material sobrenadante foi retirado e os recipientes com a ração residual colocados em estufa a 55 oC por $24 \mathrm{~h}$. A estabilidade foi calculada de acordo com a equação $\left[\left(P_{2}-P_{1}\right) / P\right] \times 100$, onde $P$ é o peso seco da amostra, $P_{1}$ é o peso dos recipientes após secagem e $P_{2}$ o peso residual da ração mais o recipiente após o tempo de imersão e secagem. A estabilidade foi expressa em porcentagem (\%) a partir do peso total inicial da ração.

\subsubsection{Determinação do teor de umidade da biomassa de A. franciscana}

O teor de água contida na biomassa congelada de $A$. franciscana foi calculado através da equação $\left[\left(P_{2}-P_{1}\right) / P\right] \times 100$, onde $P$ representa o peso úmido da biomassa descongelada e lavada com água corrente para remoção da salmoura, $P_{1}$ indica o peso do recipiente após secagem $\left(55^{\circ} \mathrm{C}\right)$ em estufa por 60 min e $P_{2}$ o peso residual da biomassa mais o recipiente após secagem na estufa a $55{ }^{\circ} \mathrm{C}$ por $24 \mathrm{~h}$. O peso da biomassa seca foi expresso em porcentagem (\%) a partir do peso total inicial da amostra úmida de $A$. franciscana.

\subsection{Análises estatísticas}

Os dados foram submetidos ao teste $\mathrm{W}$ de Shapiro - Wilk e se adequaram à distribuição normal. A Análise de Variância (ANOVA) foi aplicada para verificar a existência de variações estatisticamente significativas $(P<0,05)$ no ganho de peso absoluto $(G P A)$, no ganho de peso relativo (GPR) e na taxa de crescimento relativo (TCR) dos camarões em cada tratamento, bem como para avaliar suas respectivas taxas de consumo alimentar aparente (CAA). Em seguida, o teste de Tukey foi utilizado para examinar as diferenças estatísticas individuais entre os tratamentos, quando observadas diferenças estatísticas significativas ao nível de probabilidade de $\alpha=0,05$ (ZAR, 1996). Estes resultados foram obtidos com a utilização do Software Statistica 7.0, Startsoft 2004.

\section{RESULTADOS}

Os resultados obtidos para as variáveis abióticas (Tabela 1) mostraram que durante o período experimental os camarões permaneceram em condições adequadas ao cultivo, o que favoreceu sua sobrevivência ( $100 \%$ em todos os tratamentos dietéticos) e crescimento. $\mathrm{O} \mathrm{pH}$ variou de 7,57 a 7,95 , ao passo que a temperatura apresentou mínimo de $28,2^{\circ} \mathrm{C}$ e máximo de $29,1^{\circ} \mathrm{C}$. A salinidade apresentou mínimo de $36 \%$ e máximo de $39 \%$, enquanto que a luminosidade, registrada no período claro, variou de 565 a 1.417 lux. Por sua vez, a concentração de nitrogênio amoniacal apresentou valores entre $0,10 \mathrm{mg} / \mathrm{L}$ no tratamento com $1,8 \mathrm{mg}$ de aditivo 
alimentar (T2) e 3,27 mg/L no tratamento em que $0,6 \mathrm{mg}$ de aditivo (T1) foi incluído à ração. Ao mesmo tempo, o nitrito apresentou concentrações que variaram de $0,01 \mathrm{mg} / \mathrm{L}$ no tratamento aditivado com $1,8 \mathrm{mg}$ de $A$. franciscana (T2) ao valor máximo de $5,17 \mathrm{mg} / \mathrm{L}$ no tratamento aditivado com $3,0 \mathrm{mg}$ (T3).

Tabela 1: Valores mínimo e máximo, média e desvio padrão das variáveis abióticas pH, temperatura, salinidade, nitrogênio amoniacal $\left(\mathrm{NH}_{3}\right)$ e nitrato $\left(\mathrm{NO}_{2}{ }^{-}\right)$, registradas nos diferentes tratamentos dietéticos no decorrer do período experimental.

\begin{tabular}{|c|c|c|c|c|c|}
\hline \multirow[b]{2}{*}{ Tratamentos } & $\mathrm{pH}$ & Temperatura $\left({ }^{\circ} \mathrm{C}\right)$ & Salinidade (\%o) & $\mathrm{NH}_{3}(\mathrm{mg} / \mathrm{L})$ & $\mathrm{NO}_{2}^{-}(\mathrm{mg} / \mathrm{L})$ \\
\hline & \multicolumn{5}{|c|}{$\begin{array}{c}\text { Média } \pm \text { DP } \\
\text { (Lmin - Lmax) }\end{array}$} \\
\hline $\mathrm{T}_{1}$ & $\begin{array}{c}7,81 \pm 0,04 \\
(7,65-7,93)\end{array}$ & $\begin{array}{l}28,65 \pm 0,06 \\
(28,3-29,0)\end{array}$ & $\begin{array}{l}38,12 \pm 0,18 \\
(36,0-39,0)\end{array}$ & $\begin{array}{c}1,58 \pm 1,27 \\
(0,36-3,27)\end{array}$ & $\begin{array}{c}0,21 \pm 0,29 \\
(0,01-0,64)\end{array}$ \\
\hline $\mathrm{T}_{2}$ & $\begin{array}{l}7,76 \pm 0,09 \\
(7,68-7,95)\end{array}$ & $\begin{array}{l}28,74 \pm 0,02 \\
(27,7-29,1)\end{array}$ & $\begin{array}{l}38,35 \pm 0,19 \\
(37,0 \pm 39,0)\end{array}$ & $\begin{array}{c}0,79 \pm 0,71 \\
(0,10-1,52)\end{array}$ & $\begin{array}{c}0,52 \pm 0,04 \\
(0,01-1,92)\end{array}$ \\
\hline$T_{3}$ & $\begin{array}{c}7,80 \pm 0,02 \\
(7,71-7,95)\end{array}$ & $\begin{array}{l}28,71 \pm 0,05 \\
(28,2-29,1)\end{array}$ & $\begin{array}{l}38,28 \pm 0,11 \\
(37,0 \pm 39,0)\end{array}$ & $\begin{array}{c}1,76 \pm 1,99 \\
(0,26-4,50)\end{array}$ & $\begin{array}{c}1,32 \pm 2,57 \\
(0,01-5,17)\end{array}$ \\
\hline Controle $\left(T_{4}\right)$ & $\begin{array}{c}7,84 \pm 0,03 \\
(7,57-7,95)\end{array}$ & $\begin{array}{l}28,71 \pm 0,08 \\
(28,3-29,1)\end{array}$ & $\begin{array}{l}38,05 \pm 0,10 \\
(36,0-39,0)\end{array}$ & $\begin{array}{c}0,98-0,74 \\
(0,35-1,94)\end{array}$ & $\begin{array}{c}0,67-0,97 \\
(0,01-2,07)\end{array}$ \\
\hline
\end{tabular}

Os resultados concernentes à estabilidade física da ração comercial demonstraram que passadas 2 horas de imersão (período em que a ração ficou disponível para alimentação dos camarões), 80,9 \pm 0,39\% de seu conteúdo permaneceu íntegro em relação ao peso inicial. Por sua vez, o teste de umidade da biomassa de $A$. franciscana indicou que apenas 7,49 $\pm 0,19 \%$ de seu peso representa a massa corpórea que de fato revestiu os "peletes" da ração e, deste modo, serviu para incrementá-la com aditivo alimentar. Portanto, os valores referentes à biomassa úmida de $A$. franciscana adicionada em quantidades equivalentes a 0,6, 1,8 e 3,0 mg correspondem na verdade $0,075,0,225$ e $0,375 \%$ do peso seco da ração ofertada $\left(0,8 \mathrm{~g}_{\text {dia }}{ }^{-1}\right)$, respectivamente.

Os resultados obtidos a partir da análise do GPA e do GPR dos camarões submetidos aos diferentes tratamentos dietéticos estão sumarizados na Tabela 2. Os camarões alimentados com a ração controle (T4) obtiveram GPA de 0,33 $\pm 0,10$ g e GPR de 4,02 $\pm 1,34 \%$. Por sua vez, os indivíduos submetidos ao tratamento com $0,6 \mathrm{mg}$ de aditivo alimentar (T1) atingiram GPA e GPR de $0,79 \pm 0,32$ g e 10,07 $\pm 2,09 \%$, respectivamente. Com relação aos camarões alimentados com ração aditivada com 1,8 $\mathrm{mg}$ de $A$. franciscana (T2), o GPA foi de 1,33 $\pm 0,12 \mathrm{~g}$ (GPR de 15,39 \pm $2,06 \%$ ). Por sua vez, os animais que tiveram sua ração aditivada em 3,0 mg de $A$. franciscana (T3) obtiveram GPA e GPR iguais a 1,35 $\pm 0,35$ g e 15,60 $\pm 5,71 \%$, respectivamente. De um modo geral, o desempenho dos animais (GPA e GPR) foi significativamente melhor nos tratamentos aditivados com A. franciscana quando comparados ao grupo controle.

Tabela 2: Valores mínimo e máximo, média e desvio padrão do ganho de peso absoluto (GPA) e do ganho de peso relativo (GPR) de juvenis de Litopenaeus schmitti para os diferentes tratamentos dietéticos. 


\begin{tabular}{ccclll}
\hline \multirow{2}{*}{ Tratamentos } & \multicolumn{2}{c}{ GPA (g) } & & \multicolumn{2}{c}{ GPR (\%) } \\
\cline { 2 - 3 } \cline { 5 - 6 } & Min-Max & Média \pm DP & & Min-Max & Média \pm DP \\
\hline $\mathrm{T}_{1}$ & $0,61-1,05$ & $0,79 \pm 0,32^{\mathrm{a}}$ & & $6,99-12,82$ & $10,07 \pm 2,09^{\mathrm{a}}$ \\
$\mathrm{T}_{2}$ & $1,21-1,44$ & $1,33 \pm 0,12^{\mathrm{b}}$ & & $13,35-17,71$ & $15,39 \pm 2,06^{\mathrm{b}}$ \\
$\mathrm{T}_{3}$ & $1,04-1,35$ & $1,35 \pm 0,35^{\mathrm{b}}$ & & $8,57-22,58$ & $15,60 \pm 5,71^{\mathrm{b}}$ \\
Controle $\left(\mathrm{T}_{4}\right)$ & $0,20-0,41$ & $0,33 \pm 0,10^{\mathrm{c}}$ & & $2,29-15,38$ & $4,02 \pm 1,34^{\mathrm{c}}$ \\
\hline
\end{tabular}

Na mesma coluna, resultados seguidos por diferentes letras indicam diferenças estatisticamente significativas $(P<0,05)$.

Com relação à taxa de crescimento relativo (TCR), pôde-se verificar uma variação na média dos diferentes tratamentos, desde o valor mínimo de 0,13 $\pm 0,04 \%$.dia $^{-1}$ no grupo controle (T4) ao valor máximo de $0,48 \pm 0,06 \% \cdot$ dia $^{-1}$ e $0,48 \pm 0,17 \%$.dia ${ }^{-1}$ nos tratamentos com 1,8 (T2) e 3,0 mg (T3) de aditivo alimentar, respectivamente (Tabela 3). Foram registradas diferenças estatisticamente significativas $(P<0,05)$ entre esses tratamentos.

Tabela 3: Valores mínimo e máximo, média e desvio padrão da taxa de crescimento relativo (TCR) e do consumo alimentar aparente (CAA) dos juvenis de Litopenaeus schmitti para os diferentes tratamentos dietéticos.

\begin{tabular}{cccccc}
\hline \multirow{2}{*}{ Tratamentos } & \multicolumn{2}{c}{ TCR $\left(\%\right.$. dia $\left.^{-1}\right)$} & & \multicolumn{2}{c}{ CAA (\%BM.dia $\left.{ }^{-1}\right)$} \\
\cline { 2 - 3 } \cline { 5 - 6 } & Min-Max & Média $\pm \mathrm{DP}$ & & Min-Max & Média \pm DP \\
\hline $\mathrm{T}_{1}$ & $0,23-0,40$ & $0,32 \pm 0,06^{\mathrm{a}}$ & & $2,44-5,70$ & $3,85 \pm 1,41$ \\
$\mathrm{~T}_{2}$ & $0,42-0,54$ & $0,48 \pm 0,06^{\mathrm{b}}$ & & $3,02-3,85$ & $3,40 \pm 0,36$ \\
$\mathrm{~T}_{3}$ & $0,27-0,68$ & $0,48 \pm 0,17^{\mathrm{b}}$ & & $2,33-2,53$ & $2,96 \pm 0,43$ \\
Controle $\left(\mathrm{T}_{4}\right)$ & $0,08-0,17$ & $0,13 \pm 0,04^{\mathrm{c}}$ & & $2,90-3,70$ & $3,22 \pm 0,37$ \\
\hline
\end{tabular}

Na mesma coluna, resultados seguidos por diferentes letras indicam diferenças estatisticamente significativas $(P<0,05)$.

Por sua vez, o consumo alimentar aparente (CAA) apresentou uma variação na média desde o limite mínimo de 2,96 \pm 0,43 \%BM.dia ${ }^{-1}$ no tratamento aditivado com 3,0 mg (T3) ao limite máximo de 3,85 \pm 1,41 \%BM.dia ${ }^{-1}$ no tratamento que recebeu 0,6 mg de aditivo alimentar (T1) (Tabela 3). O grupo controle (T4) resultou em CAA com valor médio igual a 3,22 $\pm 0,37$ \%BM.dia ${ }^{-1}$. Contudo, a Análise de Variância (ANOVA) demonstrou não haver diferenças estatísticas significativas para esse critério $(P>0,05)$.

\section{DISCUSSÃO}

No presente estudo, os camarões $L$. schmitti alimentados com ração comercial aditivada com A. franciscana obtiveram, ao final do experimento, maior ganho de peso (GPR entre 10,07 e $15,60 \%$ ) do que os indivíduos do grupo controle (GPR de 4,02\%). Estes resultados assemelham-se aos de Harpaz (1997), o qual registrou um aumento de $17 \%$ no ganho de peso de juvenis de Macrobrachium rosenbergii a partir da adição de $6 \mathrm{~mL}$ de uma solução de betaína- $\mathrm{HCl}\left(10^{-3} \mathrm{M}\right)$ 
introduzida nos tanques de cultivo. Smith et al. (2005), por sua vez, comprovaram que juvenis de Penaeus monodon apresentaram ganho de peso significativamente maior com rações que continham $5 \%$ de atrativos à base de farinha de crustáceos $\left(23,01 \%\right.$.semana $\left.{ }^{-1}\right)$ ou de krill $(23,58$ \%.semana ${ }^{-1}$ ), em relação aos camarões alimentados com uma ração desprovida de atrativos. À semelhança, Córdova-Murueta \& Garcia-Carreño (2002) registraram um aumento de 34\% no ganho de peso semanal de juvenis $L$. vannamei quando estes tiveram sua ração suplementada com $3 \%$ de farinha de lula, e aumento de 31,5 e $33 \%$.semana ${ }^{-1}$ quando a ração foi aditivada com $9 \%$ de farinha de peixe e de krill, respectivamente.

O ganho de peso dos camarões no presente estudo (GPR máximo de 15,60 \%.mês ${ }^{-1}$ ), quando comparados àqueles obtidos nos trabalhos supracitados, é relativamente baixo. No entanto, é importante ressaltar que esses e outros trabalhos, que versam sobre a nutrição e eficiência alimentar de camarões, são conduzidos com dietas balanceadas para atender especificamente as necessidades nutricionais relativas às espécies que estão sendo investigadas. Fato esse que não ocorreu no presente estudo, uma vez que a ração comercial ofertada era formulada para L. vannamei. Somado a isso, sabe-se que a espécie analisada, L. schmitti, não é encontrada em cativeiro e por este motivo não está condicionada à alimentação artificial.

Os resultados também sugerem que a eficiência de $A$. franciscana como aditivo alimentar pode variar conforme o nível de inclusão, uma vez que os camarões tratados com 5\% (3,0 mg) e $3 \%(1,8 \mathrm{mg})$ de aditivo alimentar obtiveram crescimento significativamente maior $(P<0,05)$ que aqueles tratados com ração aditivada com $1 \%(0,6 \mathrm{mg})$. Esse fato leva a crer que a ração comercial ofertada aos animais foi possivelmente formulada com níveis relativamente baixos de atrativos ou que houve perdas significativas dessas substâncias durante o processamento das rações.

Contudo, à medida que se aumentava o nível de inclusão de aditivo alimentar um efeito sinérgico entre substâncias quimioatrativas contidas na $A$. franciscana (especialmente aminoácidos) e na ração provavelmente atraía os animais e estimulava o seu consumo alimentar. Essa justificativa fundamenta-se nos achados de Heinen (1980), o qual relata que atrativos alimentares são mais eficazes quando administrados em combinação do que quando fornecidos individualmente.

À semelhança dos resultados obtidos no presente estudo, Smith et al. (2005) demonstraram que o aumento do nível de inclusão de 1 para $5 \%$ de atrativos alimentares provenientes de farinha de crustáceos ou de krill numa ração composta principalmente por proteínas vegetais, provocava melhora no desempenho zootécnico de $P$. monodon. Contrariamente, Felix \& Sudharsan (2004) concluíram que o aumento do nível de 0,5 para 1,5\% de uma mistura entre o aminoácido glicina e betaína causava uma tendência a decréscimo no ganho de peso de juvenis $M$. rosenbergii; muito embora esses níveis exibissem um efeito positivo quando comparados a uma ração controle desprovida da mistura.

Apesar dos resultados obtidos para o CAA não apresentarem diferenças estatisticamente significativas, é importante mencionar a discordância encontrada entre os seus valores e àqueles obtidos para o crescimento dos camarões (GPA, GPR e TCR). São vários os trabalhos que apresentam disparidades entre o CAA e o ganho de peso dos animais. Hartari \& Briggs (1993) e Penaflorida \& Virtanen (1996), por exemplo, constataram que a inclusão de níveis mais elevados de aditivos alimentares não resultava em aumento do consumo alimentar de juvenis de $P$. monodon, muito embora melhorasse o crescimento. Do mesmo modo, Smith et al. (2005) 
demonstraram que o aumento do nível de inclusão de estimulantes alimentares, constituído por farinha de lula, numa ração para juvenis de $P$. monodon, provocava um decréscimo no consumo alimentar e um aumento no peso dos animais.

Justamente por não haver diferenças significativas no CAA entre os diferentes tratamentos, poder-se-ia imaginar que o maior crescimento dos camarões, especialmente aqueles pertencentes aos tratamentos T2 e T3, fosse devido a um maior valor nutricional da ração, conferido pela biomassa de $A$. franciscana que era incluída (3 e 5\%, respectivamente). No entanto, essa conjetura pôde ser contrariada porque os níveis de inclusão de $A$. franciscana na ração (em peso seco) constituíam quantidades ínfimas $\left(0,075 ; 0,225\right.$ e $0,375 \%$ da oferta de 0,8 g.dia $^{-1}$ de ração), o suficiente apenas para provocar a deteç̧ão e orientação do estímulo químico (dietas) e, posteriormente, o consumo.

Montemayor et al. (2005), ao verificarem a utilização de atrativos alimentares (moléculas sintéticas, extratos animais e vegetais) para juvenis de $L$. vannamei, constataram que percentuais de $0,255 \%$ e $0,242 \%$ dos aminoácidos arginina e histidina, respectivamente, foram os que desencadearam um maior grau de excitação nos animais quando comparados a outros aminoácidos testados (lisina e tirosina). Tanto esses resultados quanto os do presente estudo coincidem com o nível estabelecido como aceitável por Heinen (1980), o qual menciona que o percentual de inclusão de aditivos alimentares (especialmente aminoácidos) não deve ultrapassar $1 \%$ da dieta, devido, principalmente, aos custos das substâncias a serem adicionadas.

Os resultados obtidos para a estabilidade física da ração comercial após as $2 \mathrm{~h}$ de imersão $(80,9 \pm 0,39 \%)$ foram baixos quando comparado aos registrados por Smith et al. (2005). Estes autores demonstraram que os "peletes" de ração, contendo atrativos à base de peixe hidrolisado, após 6 h de imersão em água do mar permaneceram com 91,8\% de sua integridade física. Eles ainda concluíram que após $2 \mathrm{~h}$ de imersão, os aminoácidos lixiviaram em quantidades que variaram de 5,4 a 7,3 g. $\mathrm{Kg}^{-1}$ do total da matéria seca da ração.

Em face desses resultados, pode-se admitir que a baixa estabilidade da ração comercial utilizada no presente trabalho implicou em perdas de nutrientes essenciais, a exemplo de aminoácidos e proteínas, que poderiam ocasionar um maior crescimento dos camarões. No entanto, o método de inclusão do aditivo ("coating") empregado abrandou problemas relacionados à perda da propriedade atrativo-estimulante de $A$. franciscana, uma vez que não envolveu temperaturas elevadas durante a preparação da dieta (HARPAZ, 1997). Portanto, o revestimento com a biomassa de $A$. franciscana provavelmente ocasionou a recuperação da propriedade atrativo-estimulante da ração, favoreceu seu rápido consumo e resultou no bom desempenho dos camarões, já que a integridade nutricional da ração pôde ser mantida em virtude do curto tempo de lixiviação dos nutrientes. Esse fato justifica o porquê dos camarões tratados com a ração aditivada (T1, T2 e T3) terem obtido maior crescimento do que aqueles do grupo controle, mesmo sem haver diferenças significativas no consumo alimentar aparente (CAA).

Ao avaliarem atrativos e estimulantes alimentares para adultos de $L$. vannamei, Sanchez et al. (2005) comprovaram que passadas $2 \mathrm{~h}$ da oferta alimentar não existiram diferenças na eficácia entre um alimento aditivado com farinha de lula (5\%) e um outro desprovido de aditivo alimentar. Essa informação leva a crer que existe tendência à redução da atratividade com a progressão do tempo. Mediante isso, pode-se admitir que o período de exposição ao alimento que os camarões foram submetidos ( $2 \mathrm{~h}$ por arraçoamento ou $4 \mathrm{~h}^{\mathrm{d}} \mathrm{dia}^{-1}$ ) no presente estudo foi suficiente para 
verificar a eficácia de $A$. franciscana como aditivo alimentar. No entanto, o desempenho zootécnico dos camarões poderia ser elevado a índices ainda mais elevados se um maior fracionamento da dieta fosse aplicado, já que o tempo de exposição de cada dieta seria mantido a cada arraçoamento $(2 \mathrm{~h})$ ao passo que um maior tempo de exposição ao alimento seria proporcionado ao longo do dia.

Embora avaliar o sistema experimental não tenha sido o objetivo do presente trabalho, é relevante destacar sua importância para experimentos que examinam a nutrição de crustáceos. Além de suprimir possíveis incidências do canibalismo, o que é característico de muitas espécies de crustáceos, e eliminar influências comportamentais intra-específicas que possam deprimir o crescimento dos camarões, a estocagem individual permite registrar e quantificar várias observações que podem contribuir para aplicação de análises mais criteriosas e, consequentemente, aumentar o grau de confiança de respostas nutricionalmente induzidas. Harpaz (1997), por exemplo, em seu estudo com sistemas comunitários de cultivo atribuiu a ocorrência do canibalismo em $M$. rosenbergii, como o principal responsável pelo elevado grau de variância encontrado para os indivíduos que obtiveram as maiores taxas de crescimento.

Ainda que a utilização de sistemas experimentais como o utilizado no presente estudo contribua para um maior entendimento e confiabilidade dos resultados, é importante ressaltar que, ainda assim, a aplicação de dados provenientes dessas pesquisas não é igualmente eficiente para a prática do cultivo, uma vez que as condições de laboratório, mesmo condizentes com a sobrevida dos animais, não representam a realidade das fazendas e a complexidade de um ecossistema aquático. Não é surpreendente afirmar que a atratibilidade e/ou palatibilidade de um composto em operações industriais possa ser dissimulada por uma grande variedade de moléculas com poder igualmente atrativo presentes no substrato e na coluna de água. No entanto, convém reconhecer que é imperativo verificar a eficiência de aditivos alimentares em ensaios laboratoriais, uma vez que se tal eficiência não for comprovada por meio de significativas respostas zootécnicas, o seu uso em viveiros comerciais torna-se, em toda sua magnitude, inconsequente.

\section{CONCLUSÃO}

Finalmente, os resultados desse estudo indicam que a adição de biomassa de $A$. franciscana adulta a rações comerciais, em níveis correspondentes a 1, 3 e 5\% da oferta diária de ração, aumenta o ganho de peso de juvenis de L. schmitti. Esse efeito positivo está provavelmente vinculado à presença de substâncias potencialmente atrativas e estimulantes alimentares contidas na biomassa de $A$. franciscana.

\section{REFERÊNCIAS}

ABCC - Associação Brasileira dos Criadores de Camarão. Cultivo de camarão marinho: realidade mundial e Brasileira, mitos e verdades sobre sua sustentabilidade ambiental e contribuição para a socioeconomia das regiões de sua intervenção. (2015). http://www.abccam.com.br/site/wp-content/uploads/2015/08/Revista-ABCC-

Edi\%C3\%A7\%C3\%A3o-Especial-Agosto-de-2015.pdf. Acessado em 14 de Abril de 2016 às $02 \mathrm{~h} 22 \mathrm{~min} .2015$. 
Bueno, S. L. S. (1990). Maturation and spawning of the white shrimp Penaeus schmitti Burkenroad, 1936, under large scale rearing conditions. Journal of the World Aquaculture Society 21 (3): 170-179.

Camara, M. R. (1994). Dietary phosphatidylcholine requirements of Penaeus japonicus Bate and Penaeus vannamei Boone (Crustacea, Decapoda, Penaeidae). Ph.D. Thesis, University of Ghent, Belgium. 173p.

Camara, M. R. (2001). Dispersal of Artemia franciscana Kellogg (Crustacea; Anostraca) populations in the coastal saltworks of Rio Grande do Norte, northeastern Brazil. Hydrobiologia 466: 145-148.

Camara, M. R. (2003). Towards a sustainable Artemia industry in northeastern Brazil. In: Book of Abstracts, Aquaculture 2003, Salvador, Brazil. World Aquaculture Society, Baton Rouge, Louisiana, EUA. p. 148.

Camara, M. R., Monteiro, P. A., Reis, L. G., Costa, M. F. (2004). Farming Artemia in a multi-cycle culture system in Northeastern Brazil. World Aquaculture 35 (2): 40-42.

Carr, W. E. S. (1988). The molecular nature of chemical stimuli in the aquatic environment. In: Sensory Biology of Aquatic Animals. J. Atema, R. R. Fay, A. N. Popper \& W. N. Tavolga (Editors). pp. 3-28. Springer-Verlag, New York, NY.

Córdova-Murueta, J. H., Garcia-Carreño, F. L. (2002). Nutritive value of squid and hydrolysed protein supplement in shrimp feed. Aquaculture 210: 371-384.

Costa-Pierce, B. A., Laws E. A. (1985). Chemotactically-active feed additive for prawns (Macrobrachium rosenbergii). The Progressive Fish-Culturist 47: 59-61.

Costero, M., Meyers, S. P. (1993). Evaluation of Chemorreception by Penaeus vannamei under Experimental Conditions. The Progressive Fish-Culturist 55: 157-162.

Coutteau, P., Santos, M., Kontara, E. K., Camara, M. R. (2000). Effect of feeding attractants on feeding rate and on growing performance of penaeid shrimp. European Aquaculture Society, Special Publication 28: 153.

D’Abramo, L. R., Castell, J. D. (1997). Research Methodology. In: Crustacean Nutrition. L. R. D’Abramo, D. E. Conklin and D. M. Akiyama (Editors). pp. 3-25. World Aquaculture Society, Lousiana State University, Baton Rouge, USA.

Felix, N., Sudharsan, M. (2004). Effect of glycine betaine, a feed attractant affecting growth and feed conversion of juvenile freshwater prawn Macrobrachium rosenbergii. Aquaculture Nutrition 10: 193-197.

Harpaz, S. (1997). Enhancement of growth in juvenile freshwater prawns, Macrobrachium rosenbergii, through the use of a chemoattractant. Aquaculture 156: 221-227.

Hartari, R., Briggs, M. R. P. (1993). Effect of feeding attractants on the behaviour and performance of juvenile Penaeus monodon Fabricius. Aquaculture Fisheries Management 25: 613-624.

Heinen, J. M. (1980). Chemoreception in decapod crustacean and chemical feeding stimulants as potential fees additives. Proceedings of the World Mariculture Society 11: 319-334.

Hindley, J. P. R. (1975). The detection, location and recognition of food by juvenile banana prawns, Penaeus merguiensis de Man. Marine Behaviour Physiology 3: 193-210.

Kolkovski, S., Koven, W. M., Tandler, A. (1997). The mode of action of Artemia in enhancing utilization of microdiets by gilthead seabream Sparus aurata larvae. Aquaculture 155: 193-205. 
Lavens, P., Sorgeloos, P. (1996). Manual on the production and use of live food for aquaculture. FAO Fisheries Technical Papaer 361. Rome, FAO. 295p.

Lee, P. G., Meyers, S. P. (1996).Chemoattraction and feeding stimulation in crustaceans. Aquaculture Nutrition 2: 157-164.

Léger, P., Bengtson, D. A., Simpson, K. L., Sorgeloos, P. (1986). The use and nutritional value of Artemia as a food source. Oceanography and Marine Biology: an Annual Review 24: 521-623.

Lenz, P. H., Browne, R. A. (1991). Ecology of Artemia. In: Artemia Biology R. A. Browne, P. Sorgeloos \& C. N. A. Trotman (Editors). pp. 237-253. CRC Press, Boca Raton, Florida, EUA.

Martinez-Cordova, L. R., Campana-Torres, A., Porchas-Cornejo, M. A. (2002). The effects of variation in feed protein level on the culture of white shrimp, Litopenaeus vannamei (Boone) in low-water exchange experimental ponds. Aquaculture Research 33: 995-998.

Misamore, M., Browdy, C. L. (1997). Evaluating hybridization potential between Penaeus setiferus and Penaeus vannamei through natural mating, artificial insemination and in vitro fertilization. Aquaculture 150: 1-10.

Montemayor-Leal, J., Mendoza-Alfaro, R., Aguilera-González, C., Rodríguez-Almaraz, G. Moléculas sintéticas y extractos animales y vegetales como atractantes alimentícios para el camarón blanco Litopenaeus vannamei. Revista Aquatic 22: 1-10. http://www.revistaaquatic.com/aquatic/art.asp?t=p\&c=183. Acessado em 14 de Abril de 2016 às $02 \mathrm{~h} 22 \mathrm{~min} .2015$.

Naylor, R. L., Goldberg, R. J., Primavera, J. N., Kautsky, N., Beveridge, M. C. M., Clay, J., Folke, C., Lubchenco, J., Meoney, H., Troell, M. (2000). Effect of aquaculture on world fish supplies. Nature 405: 1017-1024.

Naylor, R. L., Willians, S. L., Strong, D. R. (2001). Aquaculture - A Gateway for Exotic Species. Science 294: 1655-1656.

New, M. B. (1976). A review of dietary studies with shrimps and prawns. Aquaculture 9: 101-144.

Nunes, A. J. P., Gesteira, T. C. V, Goddard, S. (1997). Food ingestion and assimilation by the Southern brown shrimp Penaeus subitilis under semi-intensive culture in NE Brazil. Aquaculture 149: 121-136.

Penaflorida, V., Virtanen, E. (1996). Growth, survival and feed conversion of juvenile shrimp (Penaeus monodon) fed a betaine/aminoacid additive. Israeli Journal of Aquaculture 48: 3-9.

Pittet, A., Ellis, J. Lee, P. G. (1996). Methodology for the identification and measurement of chemical stimulants for penaeid shrimp. Aquaculture Nutrition 2: 175-182.

Primavera, J. H. (1993).A critical review of shrimp pond culture in the Philippines. Reviews in Fisheries Science 1 (2): 151-201.

Rodrigues, J. (2005). Carcinocultura Marinha - Desempenho 2004. Revista da Associação Brasileira dos Criadores de Camarão (ABCC) 7 (2): 38-44.

Sanchez, D. R., Fox, J. M., Lawrence, A. L., Castille, F. L. (2005). A Methodology for Evaluation of Dietary Feeding Stimulants for the Pacific White Shrimp, Litopenaeus vannamei. Journal of the Aquaculture Society 36: 15-23.

Seidel, C. R., Kryznowek, J., Simpson, K. L. (1980). Amino acid composition and electrophoretic protein patterns of Artemia from five geographical locations. In: The brine shrimp Artemia. Vol. 
3. G. Persoone, P. Sorgeloos, O. Roels \& E. Jaspers (Editors). pp. 375-382. Universa Press, Wetteren, Bélgica.

Smith D. M., Burford, M. A., Tabrett, S. J., Irvin, S. J., Ward, L. (2002). The effect of feeding frequency on water quality of the black tiger shrimp (Penaeus monodon). Aquaculture 207: 125-136.

Smith D. M., Tabrett, S. J., Barclay, M. C., Irvin, S. J. (2005). The efficacy of ingredients included in shrimp feeds to stimulate intake. Aquaculture Nutrition 11: 263-272.

Sorgeloos, P., Lavens, P., Léger, P., Tackaert, W., Versichele, D. (1986). Manual for the culture and use of the brine shrimp Artemia in aquaculture. FAO, ARC, Gent, Bélgica. 319p.

Sorgeloos, P., Coutteau, P., Dhert, P., Merchie, G., Lavens, P. (1988). Use of brine shrimp, Artemia spp., in larval crustacean nutrition: a review. Reviews in Fisheries Science 6: 55-68.

Sorgeloos, P., Dhert, P., Candreva, P. (2001). Use of brine shrimp, Artemia spp., in marine fish larviculture. Aquaculture 200: 147-159.

Tackaert, W., Sorgeloos, P. (1991). Salt, Artemia and shrimp: integrated production of salt, Artemia and shrimp in the People's Republic of China. World Aquaculture 22 (3): 11-17.

Zar, J. H. (1990). Biostatistical Analysis. Fourth edition. Prentice-Hall. 663p.

Weston, D. P. (1991). The effects of aquaculture on indigenous biota. In: Aquaculture and Water Quality. Impacts of Aquaculture Production on Water Quality Resources. D. E. Brune and J. R. Tomasso (Editors). The World Aquaculture Society. Advances in World Aquaculture 3: 534-567. 